\title{
Corela
}

Cognition, représentation, langage

HS-34 | 2021

Les noms sous-spécifiés en français: du lexique au discours

\section{L'actualisation des noms sous-spécifiés dans le processus d'écriture enregistré en temps réel : considérations fonctionnelles}

\section{Georgeta Cislaru}

\section{(2) OpenEdition}

Journals

Édition électronique

URL : https://journals.openedition.org/corela/13850

DOI : $10.4000 /$ corela.13850

ISSN : 1638-573X

Éditeur

Cercle linguistique du Centre et de l'Ouest - CerLICO

Référence électronique

Georgeta Cislaru, «L'actualisation des noms sous-spécifiés dans le processus d'écriture enregistré en temps réel : considérations fonctionnelles », Corela [En ligne], HS-34 | 2021, mis en ligne le 09 décembre 2021, consulté le 13 décembre 2021. URL : http://journals.openedition.org/corela/13850 DOI : https://doi.org/10.4000/corela.13850

Ce document a été généré automatiquement le 13 décembre 2021.

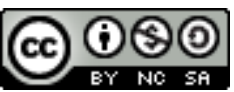

Corela - cognition, représentation, langage est mis à disposition selon les termes de la licence Creative Commons Attribution - Pas d'Utilisation Commerciale - Partage dans les Mêmes Conditions 4.0 International. 


\title{
L'actualisation des noms sous- spécifiés dans le processus d'écriture enregistré en temps réel : considérations fonctionnelles
}

\author{
Georgeta Cislaru
}

1 Comme le soulignent plusieurs travaux, les NSS constituent des objets linguistiquement complexes relativement peu explorés dans le domaine français. Mais ils constituent également des objets cognitifs dont les conditions de choix et d'actualisation n'ont jamais été observées, à notre connaissance. Or les NSS sont fonctionnellement très habiles, pouvant de ce fait assumer divers rôles au niveau de l'organisation textuelle (cf. Francis 1994, Winter 1992). Dans le présent article nous nous intéressons à l'actualisation des noms sous-spécifiés (NSS ci-après) au cours du processus d'écriture enregistré en temps réel, dans le but d'observer la mise en fonctionnement discursive de cette sous-catégorie nominale et la manière dont les NSS peuvent contribuer à structurer les dynamiques discursives. Ces observations pourraient contribuer, à terme, à une meilleure compréhension du profil sémantique des NSS.

2 Dans l'optique du passage de la langue à la parole et du virtuel à l'actuel (Bally 1965), c'est moins le point de vue du choix du déterminant (habituellement associé à la notion d'actualisation), que le choix énonciatif de produire un NSS dans un contexte donné en tant qu'émanation du lien entre pensée et langue d'une part, pensée et textualité d'autre part - qui sera au centre de nos préoccupations. Que peuvent nous apprendre les stratégies d'énonciation écrite sur la nature sémantique et le rôle textuel des NSS ? Comment ces noms varient-ils sémantiquement et fonctionnellement en dépendance du contexte d'actualisation? Une première question qui se pose est de savoir si les NSS sont actualisés spontanément dans le cadre des constructions du type NSS Être QUE P \ DE INF (voir Legallois 2008 et, pour une synthèse des constructions possibles, Schmid 2018 : 14-15). Cette observation vise, d'une part, à clarifier la portée sémantique et informationnelle des NSS en tant qu'unités de performance écrite potentiellement 
préconstruites et, d'autre part, à évaluer leur rôle dans la textualisation. Nous défendons l'idée selon laquelle les stratégies d'actualisation des NSS au cours du processus d'écriture offrent un éclairage inédit de leur fonctionnement textuel, et permettent aussi d'interroger la notion de sous-spécification, appréhendée dans la dynamique de l'actualisation online et, de ce fait, mise en relation avec la notion de complétion informationnelle. Nos hypothèses prennent appui sur une étude préalable du processus d'écriture, qui rend compte de phénomènes d'amorçage, de chunking et de prégnance de schémas constructionnels (Cislaru \& Olive 2018). Le recours à deux corpus différents, les écrits académiques et les rapports éducatifs, nous permettra par ailleurs de tester la dépendance fonctionnelle des NSS des contraintes de genre.

3 La première section pose quelques jalons définitionnels des NSS tandis que la section deux décrit les particularités du corpus écrit recueilli en temps réel et discute de la pertinence de la pause comme marqueur sémantique et cognitif possible. Les sections trois et quatre se penchent respectivement sur le relevé des occurrences de NSS et des constructions qui les hébergent, et sur l'évaluation du rôle des NSS dans le processus de textualisation.

\section{Les NSS, caractéristiques définitoires et identification}

4 Les NSS se définissent comme une catégorie fonctionnelle, qui se révèle dans l'usage (cf. Legallois 2008). Mais leur cadre fonctionnel est envisagé sous divers angles et le principe fonctionnel peut apparaître comme nécessaire mais non suffisant. La désignation même de cette catégorie nominale est symptomatique des points de vue mis en évidence par différents auteurs. Par exemple, NSS et shell nouns mettent en avant les attentes sémantiques et informationnelles qui les caractérisent (voir les travaux de Schmid), labels renvoie à leur rôle dans l'installation de catégories (voir les travaux de Francis), tandis que signalling nouns (voir Flowerdew et Forest 2015) insiste sur leur dimension projective. Nous ne retiendrons dans cette section que les concepts effectivement opératoires dans les limites de notre étude, sans prétendre formuler une définition exhaustive des NSS, une telle ambition dépassant les objectifs fixés.

\subsection{Aspects sémantiques et informationnels}

5 La définition de ces noms en tant que sous-spécifiés soulève la question de leur traitement sémantique. En effet, les avis divergent à ce sujet. Des auteurs comme Flowerdew et Forest (2015), Roze et al. (2014 : 377) parlent d'incomplétude sémantique :

This leads us to our primary discourse semantic criterion for the identification of an SN in our corpus. The relationship between an SN and its lexical realisation must construe the semantics of a clause or sequence of clauses. The construed clause need not be finite. It may also consist of a nominalisation. But an agnate clause or text semantics must be identifiable. (Flowerdew et Forest $2015: 40$ )

6 En revanche, Legallois (2008: 116) considère qu'il ne s'agit pas de non-spécification sémantique à proprement parler, mais de non-spécification informationnelle.

De ce fait, la non spécification n'est pas, à proprement parler, sémantique, comme le dit Schmid (et généralement les travaux anglo-saxons), mais, plus exactement, informationnelle. Il s'agit bien, pour le NSS, de se grossir du contenu informationnel véhiculé par la partie spécificationnelle. (Legallois 2008 : 114) 
7 D'autres travaux adoptent une position plus nuancée, en faisant la différence entre les noms sous-déterminés sémantiquement et les noms ayant un sens plein par ailleurs mais pouvant être employés en tant que NSS, en mettant l'accent sur la dénotation (Adler \& Legallois $2018: 4$ ).

8 Nous retenons comme critère définitionnel général la nécessité de spécification par le contexte du nom, qui est sémantiquement et/ou informationnellement sous-déterminé. Cela implique, selon plusieurs auteurs s'inscrivant dans le cadre des grammaires constructionnelles, l'occurrence dans des constructions spécifiques (Schmid 2000, et plus particulièrement Legallois 2006, 2008), où la caractéristique des NSS est d'être positionnés en attente d'une spécification. Cette attente de spécification devrait être d'autant plus saillante dans la dynamique du processus d'écriture, où il devient possible d'observer la manière dont la spécification prend forme, littéralement.

\subsection{Encapsulation et étiquetage textuel}

9 La nécessité de spécification implique de fait que le NSS encapsule le contenu sémantique et informationnel qui le spécifie. Legallois (2008:125) utilise le terme d'emballage comme équivalent d'encapsulation, et considère ce phénomène comme la contrepartie cognitive de la spécification. Élaboré dans le cadre de la linguistique de corpus, le principe d'encapsulation est formulé par Sinclair (voir 1993: 7). Selon ce principe, tout énoncé encapsule le précédent par un acte de référence ; en se référant à l'ensemble de l'énoncé qui précède, le nouvel énoncé l'utilise comme une partie de l'objet du discours, ce qui a pour effet d'annuler la fonction discursive, relative à un état figé du texte, pour n'en conserver que le sens, inscrit dans une dynamique de construction-reconstruction. On a donc affaire à un changement qualitatif qui est à la base du chunking et du traitement linéaire de l'information.

Pour Schmid (2000:12), la notion d'encapsulation rend compte de son idée que les NSS mettent en place des frontières conceptuelles: "shell nouns create conceptual boundaries by casting larger chunks of information into nominal structures" ${ }^{1}$. Chez ces auteurs, l'encapsulation est un critère définitoire central (au sens de critère équatif) des NSS.

11 Flowerdew et Forest (2015:5) utilisent le terme d'encapsulation pour renvoyer au principe de substitution et aux indices d'interprétation du discours (comme un fait, une possibilité, une idée, etc.)

In discourse, SNs 'stand in' for the kinds of complex notions typically expressed in full sentences and even paragraphs. By standing in for these complex notions, SNs help create and maintain continuity in text. (Flowerdew et Forest $2015: 2$ )

12 Ce point de vue se rapproche de celui de Francis (1994), qui parle de labelling en référence à la capacité des noms d'assurer des fonctions de liage et d'organisation textuelle, que ce soit de manière progressive ou rétrospective.

On constate que la capacité d'encapsulation contribue largement à la gestion de la cohérence-cohésion du texte, alors que la référence des NSS est endophorique (i.e. réfèrent au texte lui-même). Condensation, étiquettes, labels, ce sont quelques termes parmi ceux couramment utilisés. Le versant grammatical des NSS, résultat d'une sorte de grammaticalisation au sens de la métaphore grammaticale de Hallyday, leur confère 
ainsi un statut privilégié dans la gestion de la textualité, qu'Adler et Moline (2018:6) situent à la jonction entre cohésion lexicale et cohésion grammaticale.

Que ce soit dans l'optique de la schématisation de structures textuelles de base, comme Situation-Évaluation, ou dans l'optique de la linéarité textuelle (expectancy/prediction entraînant une réalisation lexicale chez Winter 1977, prospection chez Francis 1986 ou chez Sinclair et Mauranen 2006), les NSS apparaissent donc comme des indices de textualisation pertinents pour l'analyse des productions écrites enregistrées en temps réel.

\subsection{Subjectivité et intersubjectivité}

La plupart des travaux reconnaissent le rôle métadiscursif des NSS, sortes de marqueurs énonciatifs proposant des catégories évaluatives, à l'instar de Schmid (2000), qui distingue entre les noms factuels (objectifs), modaux (possibilité), événementiels (action) et circonstantiels (temps) ; ou encore de Jiang et Hyland (2015: 535), qui identifient les NSS en tant que stance nouns dont ils proposent une classification. Adler et Legallois (2018:6) distinguent, après les fonctions idéationnelle et textuelle, la fonction interpersonnelle qui se donne "le plus souvent sous un mode argumentatif, notamment grâce à la valeur modale du nom"2. Il est clair que le choix de l'énonciateur d'étiqueter un contenu sémantico-informationnel plutôt par fait ou plutôt par problème révèle un positionnement subjectif qui donne des clés interprétatives au co-énonciateur mais qui impose, par ailleurs, un point de vue difficilement remis en cause, grâce à la présupposition existentielle de la nominalisation. Les valeurs qui sont associées aux noms ne relèvent cependant pas d'un choix ponctuel, comme le soulignent Jiang et Hyland (2015: 530) : "Stance, in other words, is not simply a personal take on something, a position towards a claim or finding, but simultaneously taps into and represents a community's system of values". La nature dialogique des NSS fait qu'ils renvoient à "une étape sémiotico-cognitive récurrente dans la diversité des expériences et partagées par tous" (Legallois 2008: 123).

16 Le système de valeurs partagé semble entrer quelque part en contradiction avec la nécessité de complétion informationnelle des NSS, ce qui est partagé pouvant rester implicite. Mais c'est sans compter sur deux aspects: d'une part, le traitement occurrentiel des NSS, qui sont réfractaires à toute subordination notionelle de type hyperonymique, ce qui justifie l'apport informationnel et, d'autre part, leur fonctionnement endophorique, qui en fait des outils d'organisation textuelle, ce qui signifie que les NSS sont hybrides, en emploi à la fois référentiel et métadiscursif/ métalinguistique, chaque occurrence revenant à présupposer une relation appellative (on appelle cela un problème; ou, plutôt, on va appeler cela 'un problème'). Cette relation peut être préconstruite ou $a d$ hoc ; lorsqu'elle est préconstruite - ce qui peut arriver très souvent, on le suppose - on peut compter sur le "pouvoir phraséologique" (Adler et Moline 2018 : 14) des NSS et sur les effets de la mémorisation des structures discursives. Ce dernier point est également crucial pour notre recherche, dans la mesure où nous avançons l'hypothèse que les séquences mémorisées - ici, les constructions NSS - sont actualisées de manière spontanée et sans marquage pausal significatif dans le processus de textualisation enregistré en temps réel. 


\section{Données et méthode}

17 Le processus d'écriture est consigné en temps réel grâce à des logiciels de suivi des frappes (cf. Inputlog, Leijten et Van Waes 2006), qui enregistrent les frappes au clavier, les mouvements de souris, la durée des pauses et la durée de production de séquences langagières, en permettant ainsi de dégager des unités linguistiques de performance écrite.

\subsection{Corpus et présentation des données}

D'un point de vue comportemental, la rédaction d'un texte peut se décrire de la façon suivante: les scripteurs alternent des moments de pauses, sans écriture, avec des moments de transcription continue du texte, pendant lesquels le rédacteur produit de façon ininterrompue des séquences textuelles, que nous appelons des "jets textuels " (le terme original anglais est "burst", Chenoweth et Hayes 2001). Le jet textuel constituera notre unité d'analyse.

Les jets textuels peuvent prendre la forme d'une lettre, d'un mot, ou d'une séquence de mots - ils ne correspondent donc pas à une catégorie morphosyntaxique bien délimitée. Par exemple, l'énoncé une cousine qui peut venir partager du temps avec elle pendant le week-end a été produit sous la forme suivante dans un rapport éducatif:

[pause] une cousine qui [pause] peut venir partager du temps avec elle pendant [pause] le [pause] $w$ [pause] eek [pause] - [pause] end. [pause]

Ces hétérogénéités morphosyntaxiques des jets textuels peuvent être compensées par des régularités sémantiques et cognitives (voir 2.2.).

21 Relevant des données comportementales, la segmentation pausale bénéficie d'une vision périphérique dans le champ de la linguistique de l'oral (cf. Hirsch et Dodane 2018), tandis que la segmentation pausale à l'écrit est quasi-absente des études linguistiques. Il faut se tourner alors vers la psychologie cognitive ou la psycholinguistique, qui distinguent entre les pauses purement techniques (situées en général en-dessous d'un seuil donné : 2, 3 ou 4 secondes, par exemple), et les pauses présentant un intérêt cognitif, dans la mesure où elles permettraient de planifier la ou les séquences à venir. Avec un seuil de pause fixé à 2 secondes, ce sont les pauses à valeur cognitive que nous retenons pour notre étude (voir Olive 2014, Cislaru et Olive 2018 pour une synthèse), en formulant l'hypothèse que l'emplacement de la pause visà-vis des NSS est potentiellement un indice des dynamiques d'organisation textuelle.

Notre étude porte sur un corpus de rapports éducatifs de la protection de l'enfance et d'écrits académiques. Nous avons observé l'actualisation en temps réel des NSS en corrélant leur rôle dans le processus de textualisation et les particularités de segmentation pausale. Nous avons distingué les types de NSS et plus particulièrement leur répartition dans chacun des genres discursifs observés, catégorisé les occurrences de NSS selon leur fonctionnement anaphorique ou cataphorique, évalué leur apport discursif et métadiscursif en distinguant deux rôles: i) signaler l'organisation du discours et ii) signaler un point de vue sur l'organisation du discours. 


\subsection{La segmentation pausale et ses régularités dans les corpus}

Dans les deux corpus étudiés, on observe une série de régularités pausales qui pourraient être interprétées comme des marqueurs de relations spécifiques entre les unités linguistiques. Cislaru et Olive (2018) notent plusieurs régularités situant la pause devant des unités demandant un effort cognitif particulier, comme les choix lexicaux délicats (noms propres, termes axiologiquement marqués, etc.), l'actualisation des anaphores résumantes qui impliquent une opération d'empaquetage avant, ou l'introduction d'une séquence de discours autre, qui signale une rupture énonciative.

Ce dernier cas de figure trouve une illustration particulière dans les dossiers académiques des étudiants, où une pause significative est marquée avant l'introduction d'un concept ou d'un point de vue :

$(1)^{3}$ JM Abram penche pour « [P]

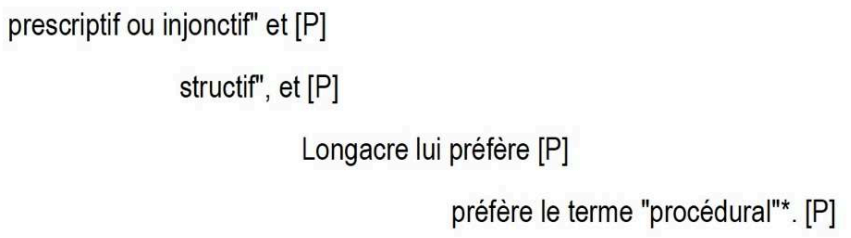

Enfin, des régularités caractéristiques des rapports éducatifs mais attestées également dans les dossiers académiques mettent au jour une tendance à marquer une pause avant l'introduction d'une spécification - sur le modèle SUJET est/a+ :

(2) [Enfant] a pu dire que c'était...[P]

[Enfant] bénéficie d'une therapie... [P]

[Enfant] ne veut pas perdre...[P]

[Enfant] nous parait dans ces moments...[P]

[Enfant] se prétend...[P]

Les régularités observées semblent pointer des relations privilégiées entre la segmentation pausale et les caractéristiques sémantico-informationnelles des séquences langagières actualisées après la pause.

Il est supposé que les NSS bénéficient d'une facilité d'actualisation et ne demandent pas un effort cognitif important, à la différence des éléments qui viendraient les spécifier. Les régularités observées sur l'ensemble du corpus, avec des pauses intervenant entre une séquence à portée générale, souvent schématisable d'un point de vue constructionnel, et une séquence à valeur spécificationnelle, comme dans Sujet + verbe avoir/être [pause] spécification (voir l'exemple (2)), constituent une piste privilégiée pour aborder la question des NSS dans l'écriture enregistrée en temps réel. L'hypothèse est que les NSS fonctionnent en projection, leur actualisation suivant la même logique, avec une segmentation pausale entre le NSS et la séquence à valeur spécificationnelle. 


\title{
3. Actualisation des NSS dans le temps réel de l'écriture
}

\begin{abstract}
nous avons fait un relevé du lexique (3.1.), des constructions NSS dans nos corpus (3.2.)
\end{abstract} et des régularités de segmentation pausale (3.3.).

\subsection{Relevé des NSS}

Dans le cadre de ce travail, l'analyse des NSS prend pour point de départ les listes de noms sous-spécifiés et schémas constructionnels inspirés par Schmid (2000), Legallois (2008), Roze et al. (2014), Flowerdew et Forest (2015). Nous avons relevé les occurrences de NSS et validé leur fonctionnement en tant que NSS par la présence d'un des schémas constructionnels appropriés ou par un test d'identification spécificationnelle en cas d'ambiguiité liée à la spécificité des données recueillies en temps réel ${ }^{4}$.

\begin{tabular}{|c|c|c|}
\hline & Dossiers académiques & Rapports éducatifs \\
\hline $\begin{array}{l}\text { Nombre } \\
\text { d'occurrences } \\
\text { recueillies }\end{array}$ & 53 & 35 \\
\hline $\begin{array}{l}\text { Nombre } \\
\text { d'occurrences } \\
\text { validées }\end{array}$ & 49 & 32 \\
\hline $\begin{array}{l}\text { Tokens } \\
\text { relevés }\end{array}$ & $\begin{array}{l}\text { 19 } \\
\text { aspect, but, catégorisation, concept, } \\
\text { connaissance, effet, exemple, fait, finalité, } \\
\text { fonction, idée, impression, intérêt, objectif, objet, } \\
\text { preuve, problème, question, registre, visée }\end{array}$ & $\begin{array}{l}\text { décision, effets, fait, fonction, idée, impression, } \\
\text { moyen, nécessité, objectif, problème, projet, } \\
\text { question, sentiment, souci }\end{array}$ \\
\hline $\begin{array}{l}\text { Tokens les } \\
\text { plus } \\
\text { représentatifs }\end{array}$ & but*, aspect, finalité, fonction, impression & question*, impression, nécessité, objectif \\
\hline
\end{tabular}

Tableau 1. Relevé des NSS dans les corpus.

* Les noms en gras sont les NSS les plus fréquents dans les corpus respectifs.

Les NSS communs aux deux corpus sont, par ordre alphabétique : effet(s), fait, fonction, idée, impression, objectif, problème, question.

On constate, de manière assez prévisible, que chaque genre discursif exploite ses propres listes de NSS. Parmi les termes les plus fréquents, des sélections lexicales spécifiques s'opèrent au sein du même champ sémantique : ainsi but et finalité sont préférés en emploi sous-spécifié dans les dossiers académiques, et objectif est préféré dans les rapports éducatifs.

Dans le temps réel de l'écriture, le NSS lui-même peut faire l'objet d'un retravail lexical, comme le montre l'exemple

(3)

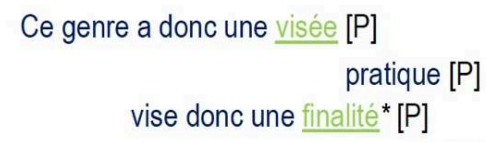

* vise donc une finalité remplace ici a donc une visée 

nominale en général. On constate que les noms, toutes sous-catégories confondues, peuvent apparaitre aussi bien en borne droite d'un jet textuel, avant la pause, qu'en borne gauche, après la pause. Par exemple, dans les dossiers académiques, environ 500 occurrences des noms sont actualisées en tant que borne droite (dans le champ [pause] littéraire) et environ 550 occurrences sont actualisées en tant que borne gauche ([pause] Welrich la nomme [pause] ui attribue le terme "inonctif-instructionnel").

Les NSS ne suivent pas cette répartition des segmentations pausales propre à la classe nominale, la pause étant marquée notamment après l'actualisation du nom et avant la formulation du contenu appelé à le spécifier. Cependant, une pause peut également être marquée avant les NSS, et ce dans les deux corpus: ex. projet, résultats, demande, 
comportement (RE) ; réalisation, engagement, attentes (DA). Dans les pages qui suivent, nous tenterons une explication fonctionnelle de ces principes de segmentation.

\section{Les NSS dans le processus de textualisation: segmentation pausale et connectivité informationnelle}

En nous inspirant des travaux de M. Charolles (2005) et de la dimension linéaire des relations d'organisation textuelle, particulièrement en phase avec une approche dynamique de l'écriture enregistrée, nous distinguerons la connexion, qui représente des relations ascendantes (backwards), de l'indexation, qui représente des relations descendantes (forward labelling) ${ }^{7}$. C'est par rapport à ces deux types de relations que nous évaluerons les conditions d'actualisation des NSS et la segmentation pausale.

\subsection{Indexation : segmentation pausale après les NSS en projection}

D'une manière générale, la segmentation pausale a tendance à se réaliser juste après le NSS et avant la spécification qui lui succède immédiatement; il s'agit de relations d'indexation. Ces NSS fonctionnent en projection, introduisant une attente en matière de complétion informationnelle et amorçant la suite de l'énoncé, comme on peut le voir dans les exemples (4) et (5) avec la construction NSS (est) de X et l'exemple (6), avec la construction NSS que X. Le NSS lui-même peut être actualisé au sein d'une séquence plus longue (ex. 4) ou seul/accompagné de son déterminant (ex. 6-7) :

(4)

Elle a effectué quelques stages au court de l'année avec pour objectif [P]

$$
\text { de définir }[\mathrm{P}]
$$

quelle entreprise serait le mieux adaptée à ses difficultés [P]

(5)

le groupe. Notre souci est d' [P]

est d'éviter une exclusion du jeune [P]

en lien avec un acte grave $[\mathrm{P}]$

(6)

. Il pr [P]

écise également $[\mathrm{P}]$

la nécessité $[P]$

que Kathy puisse effectuer un stage en ESAT [P]

Lorsque le NSS déclenche une énumération (ex. 7), il a également un fonctionnement projectif : 
(7)

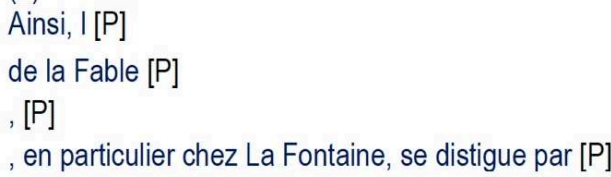

$$
\text { ce double }[P]
$$

aspect $[P]$

: la brièveté et la clarté, la soustraction et l'évidence. [P]

Comme on peut le voir dans les exemples ci-dessus, les frontières pausales ne sont pas nettement tranchées, mais la pause avant la spécification reste une constante. Les cas de figure où le NSS est séparé du segment le spécifiant par une incise (en grisé ici) sont instructifs à ce sujet, dans la mesure où ils permettent de confirmer que, indépendamment de la manière dont est actualisé le NSS lui-même (autonome ou au sein d'une séquence plus longue), la pause est constamment marquée avant la spécification informationnelle du NSS (ex. 8) :

(8)

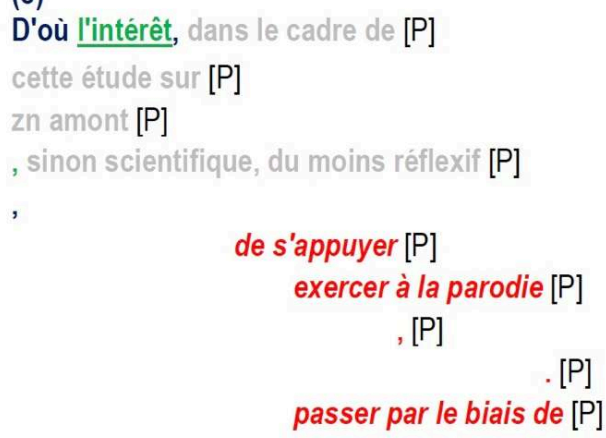

Ces données semblent confirmer l'hypothèse selon laquelle les NSS en projection fonctionnent comme des signaux (ici le terme de signalling nouns conviendrait très bien) qui projettent des attentes de complétion informationnelle d'un certain type, mais que le contenu informationnel lui-même nécessite un effort cognitif suffisamment important avant d'être actualisé, ce qui provoque une segmentation pausale entre le NSS et la séquence spécificationnelle qu'il indexe.

\subsection{Connexion et déjà-là informationnel : segmentation pausale avant un NSS}

Plus rarement, la segmentation pausale peut intervenir juste avant un NSS ; il s'agit de relations de connexion. Si l'on observe de près des noms comme difficulté ou problème, qui occupent une place importante dans les rapports éducatifs (cf. Veniard 2008), on constate que la pause intervient avant le NSS lorsque ces noms fonctionnent en tant qu'anaphores résumantes; cela correspond aux régularités observées dans les corpus enregistrés en temps réel pour l'actualisation des reprises résumantes autres que les NSS (démonstratifs, etc.).

Dans l'exemple (9), nous soulignons en italique l'antécédent de deux NSS en construction résomptive. Comme dans d'autres configurations, cet antécédent peut 
être plus ou moins développé, mais il comprend toujours au moins un prédicat. La pause marque une sorte de clôture de l'information à résumer, avant l'actualisation effective du NSS résumant. C'est donc le cas précis des NSS bénéficiant d'une spécification en amont qui est considéré ici. On peut supposer que l'actualisation de ces noms nécessite un effort cognitif plus important, et qu'il sont le résultat d'un choix lexical réfléchi :

(9)

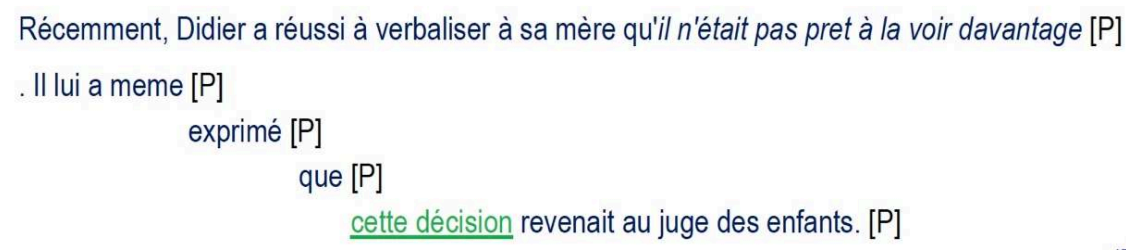

Il convient cependant de nuancer le propos, dans la mesure où, pour des raisons inhérentes aux dynamiques cognitives du processus d'écriture (longueur limitée des séquences pouvant être produites en un seul jet, multiplicité et complexité des phénomènes langagiers intervenant au niveau de la production d'un jet textuel, au-delà de la relation résomptive, etc.), l'interprétation des pauses n'est pas univoque. On ne peut pas considérer que la pause est déterminée par le seul fait de l'emploi résomptif du NSS. La segmentation informationnelle répond à plusieurs critères qui peuvent converger ou diverger, les NSS étant employés dans des contextes complexes où plusieurs paramètres entrent en ligne de compte. Ainsi, dans le cas de ce dernier exemple (9), la segmentation avant le NSS peut être doublement déterminée par le fonctionnement résomptif de ce dernier et par la frontière entre discours cité et discours citant, souvent marqué par une pause longue, comme cela a pu être noté par Cislaru et Olive (2018).

Dans un autre ordre d'idées, lorsqu'ils sont modifiés par un complément et constituent donc des descriptions définies où le nom est le classificateur de la valeur qui le spécifie (cf. Legallois 2008), les NSS sont actualisés dans la même séquence que leur spécification, sans pause entre les deux : ce dernier ne réussit pas à l'apaiser complètement ,en témoigne [sic] ses difficultés à se mobiliser scolairement. (RE).

Les autres cas de figure où la pause est marquée avant le NSS et ne sépare plus ce dernier de sa spécification mettent en évidence des différences de traitement cognitif s'opposant au fonctionnement en projection. Ainsi, il s'agit toujours de contextes dans lesquels la spécification du NSS n'a pas à être produite de manière ad hoc, mais est reproduite à partir d'un stock de connaissances déposées dans la mémoire à moyen ou long terme. Plusieurs marqueurs linguistiques permettent d'appuyer cette hypothèse : les marqueurs polyphoniques, les marques du passé, etc. Bien qu'hétérogènes d'un point de vue grammatical et même énonciatif, ces marqueurs partagent la même dimension cognitive-informationnelle : ils se rapportent à des informations et données ayant fait l'objet d'un pré-traitement au moment de l'actualisation écrite des NSS. Ainsi, par exemple, les marqueurs polyphoniques renvoient à du discours déjà-là, tandis que les marques du passé renvoient à des procès antérieurs au moment de l'énonciation et donc traités en tant que tels avant l'actualisation.

Dans l'exemple (10), le NSS est emboîté en site polyphonique, le scripteur se positionnant par rapport à un type de connaissance en particulier. Alors que dans les 
emplois projectifs des NSS cités ci-dessus le positionnement du scripteur concerne la spécification elle-même, pour laquelle il propose une classification ad hoc, dans les exemples étudiés ici c'est la construction NSS dans son ensemble (NSS+spécification) qui est présentée comme un savoir partagé.

(10)

Seulement ici, $[\mathrm{P}]$

sans la connaissance qu'il sagit du journal en réalité [P]

, Kitty peut représenter un réel intelocuteur, ce que d'ailleur aurait aimé Anne Frank [P]

en lui donnant ce prénom. [P]

Il en est de même dans les constructions formulées au passé, ce temps verbal étant le marqueur d'un déjà-là informationnel (du moins pour le scripteur) réactualisé dans le site discursif.

(11)

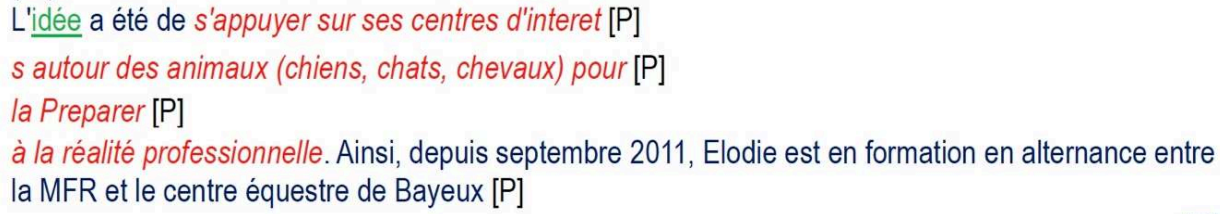

Un dernier cas de figure est celui de fait, qui est reconnu comme une exception dans le champ des NSS (cf. Huyghe $2018^{8}$; voir aussi Jiang et Hyland 2015 pour l'anglais fact). Dans les exemples recueillis, l'emploi de fait est attesté dans des contextes où le contenu actualisé - i.e., la séquence que l'on pourrait considérer comme la spécification du NSS - peut difficilement être nominalisé et catégorisé de manière stable par un nom morpho-sémantiquement lié (voir notamment l'exemple 12). Le nom n'y est pas anaphorique mais joue néanmoins un rôle important dans la textualisation. En effet, l'emploi de fait est le résultat d'un priming contextuel, les séquences soulignées qui le précèdent immédiatement projetant l'attente d'une spécification ou d'une reformulation.

II était donc partagé entre [P]

$$
\text { la protection de ses soeurs et [P] }
$$

le fait de ne plus les voir aussi souvent, [P]

(13)

Ce sont dans ces moments qu'Antonys se livre le plus [P]

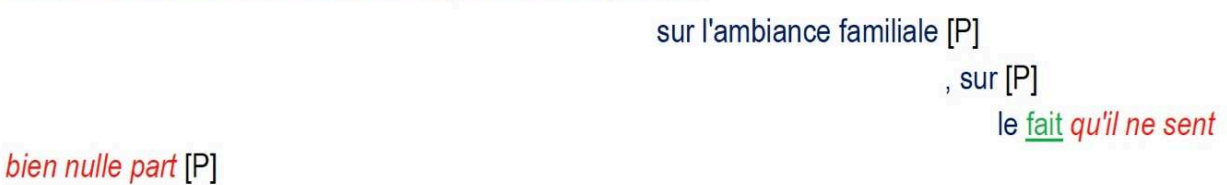

Enfin, étant donné la nature des formulations, toutes les deux tirées de rapports éducatifs, il est probable qu'il s'agisse d'une reprise des paroles de l'enfant - ce qui justifierait une segmentation pausale avant une séquence de discours autre, comme dans l'exemple (9). Cela expliquerait également le choix du nom fait, qui n'implique pas 
de positionnement axiologique (Schmid 2000) et qui permet donc au scripteur de rester le plus neutre possible, comme exigé par les guides de rédaction des rapports éducatifs.

\section{Conclusions} entre les opérations de connexion et d'indexation réalisées par les NSS. Nous avons noté des degrés de solidarité différents, les conditions d'actualisation des NSS variant selon le fonctionnement de ces derniers : en projection, résomptifs ou citationnels, polyphoniques, etc. Au niveau sémantico-cognitif, on pourra ainsi distinguer entre les catégorisations ad hoc, où le NSS peut être considéré comme un hyperonyme procédural - il est alors isolé de sa spécification par une pause -, et le fonctionnement idiomatique où le nom, spécifié, désigne un «sous-type de ", qui tend à induire deux modes d'actualisation différents dans le temps réel de l'écriture - l'actualisation est unitaire, sans pause significative entre le NSS et sa spécification. Dans ce dernier cas, les NSS fonctionnent comme des éléments de schémas généraux préconstruits; on a affaire à des routines installant des jalons argumentatifs au niveau structurel ou informationnel. La segmentation pausale avant un NSS à fonction résomptive - relevant de la connexion - confirme le processus de chunking incrémental, en marquant une frontière entre une information spécifiante et l'opération d'encapsulation par le NSS, qui indique un changement qualitatif.

58 Finalement, une des questions qui se pose est de savoir si un NSS, défini d'un point de vue fonctionnel, doit nécessairement être en attente d'un contenu spécificationnel qui ne serait pas "déjà-là ", et qui serait toujours en cours d'élaboration au moment de l'actualisation du NSS - le NSS prototypique serait alors le NSS en projection, réalisant une opération d'indexation textuelle. 


\section{BIBLIOGRAPHIE}

Adler, S. \& Legallois, D. (2018). « Les noms sous-spécifiés dans le débat parlementaire : analyse fréquentielle et catégorisation modale ». Langue française 198, 19-34.

Adler, S. \& Moline, E. (2018). « Les noms généraux : présentation ». Langue française 198, 5-18. Bally, Ch. (1965 [1932]). Linguistique générale et linguistique française. Paris : Librairie Ernest Leroux. Charolles, M. (2005). « Framing adverbials and their role in discourse cohesion : from connexion to forward labelling ». Papers of the Symposium on the Exploration and Modelling of Meaning (Sem 05), Biarritz, 14-15 novembre 2005. (http://www.univ-paris3.fr/m-charolles-michel-32163.kjsp)

Chenoweth, N. A. \& Hayes, J. R. (2001). « Fluency in Writing : generating text in L1 and L2 ». Written Communication 18 (1), 80-98.

Cislaru, G. \& Olive, Th. (2018). Le processus de textualisation. Bruxelles : De Boeck.

Hirsch, F. \& Dodane, C. (éds). (2018). « Organisation spatiale et temporelle des pauses en parole et en discours ». Langages 211.

Flowerdew, J. \& Forest, R. (2015). Signalling Nouns in English: A Corpus-based Discourse Approach. Cambridge : Cambridge University Press.

Francis, G. (1986). Anaphoric Nouns. Discourse Analysis Monographs 11. University of Birmingham.

Francis, G. (1994). « Labelling Discourse: An Aspect of Nominal-Group Lexical Cohesion ». In M. Coulthard (éd.), Advances in Written Text Analysis. London - New York, Routledge, 83-101.

Huyghe, R. (2018). « Généralité sémantique et portage propositionnel : le cas de fait ». Langue française 198, 35-50.

Jiang, F. (K.) \& Hyland, K. (2015). « 'The fact that': Stance nouns in disciplinary writing ». Discourse Studies 17(5), 529-550.

Legallois, D. (2006). «Quand le texte signale sa structure : la fonction textuelle d'une certaine catégorie nominale ». Corela $\mathrm{n}^{\circ}$ spécial « Cohésion, Cohérence et Textualisation ». (https:// corela.revues.org/1465).

Legallois, D. (2008). « Sur quelques caractéristiques des noms sous-spécifiés ». Scolia 23, 109-127.

Legallois, D. \& Vajnovszky, A. (ce volume).

Leijten, M. \& Van Waes, L. (2006). «Inputlog : New Perspectives on the logging of on-Line writing ». In G. Rijlaarsdam (Series éd.) Studies in Writing, Vol. 18, and K. P. H. Sullivan \& E. Lindgren (Vol. éds), Computer Keystroke Logging and Writing : Methods and Applications. Amsterdam : Elsevier, 73-94.

Olive, Th. (2014). « Toward an Incremental and Cascading Model of Writing : A review of research on writing processes coordination ». Journal of Writing Research 6, 173-194.

Roze, C., Charnois, Th., Legallois, D., Ferrari, S. \& Salles, M. (2014). « Identification des noms sousspécifiés, signaux de l'organisation discursive ». 21ème Traitement Automatique des Langues Naturelles, Marseille, 377-388.

Schmid, H.-J. (2000). English Abstract Nouns as Conceptual Schells. Berlin - New York : Mouton de Gruyter. 
Schmid, H.-J. (2018). « Shell nouns in English - a personal roundup ». Caplletra. Revista Internacional de Filologia 64, 109-128.

Sinclair, J. M. (1993). « Written discourse structure ». In J. M. Sinclair, M. Hoey \& G. Fox (éds), Techniques of Description. Spoken and written discourse. A festschrift for Malcolm Coulthard. LondonNew York : Routledge, 6-31.

Sinclair, J. M. \& Mauranen, A. (2006). Linear Unit Grammar : integrating speech and writing. Amsterdam - Philadelphia : John Benjamins.

Veniard, M. (2008). «Écrire "ce qui ne va pas" dans le champ de l'enfance en danger : les mots problème(s) et difficulté(s) ». Les Carnets du Cediscor 10, 57-77.

Winter, E. (1977). « A Clause-Relational Approach to English Texts: A Study of Some Predictive Lexical Items in Written Discourse ». Instructional Science 6, 1-92, Amsterdam : Elsevier.

Winter, E. (1992). « The Notion of Unspecific Versus Specific as One Way of Analysing The Information of a Fund-Raising Letter ». In W. C. Mann \& S. A. Thompson (éds), Discourse Description: Diverse Linguistic Analyses of a Fund-raising Text. Amsterdam : John Benjamins, 131-70.

\section{NOTES}

1. D'où aussi son choix terminologique, comme il l'explique plus loin : "Looking at the collection of terms and the different images underlying such notions as containment, signalling, pointing and encapsulating, I think that with a little stretch of imagination the shell metaphor incorporates all these aspects." (Schmid 2000 : 13)

2. Sur les valeurs modales des NSS, voir D. Legallois et A. Vajnovzsky ici-même.

3. Tous les exemples extraits de nos corpus sont anonymés mais reproduits sans aucune correction orthographique ou grammaticale. Chaque saut de ligne implique une pause d'au moins 2 secondes, notée $[\mathrm{P}]$ - une ligne correspond donc à un jet textuel. Lorsque la mise en page le permet, nous rendons compte de la linéarité de l'écriture, en faisant figurer chaque séquence produite après une pause d'au moins 2 secondes en dessous de la séquence précédente (sur l'axe vertical), mais dans sa continuité de celle-ci sur l'axe horizontal (voir l'exemple (6)).

En cas de reformulation au fil de l'écriture, un fragment prend la place du précédent: voir l'exemple (3), où [vise donc une finalité] ligne trois vient se substituer à [a donc une visée] ligne une. Dans les cas où les jets textuels contenant des NSS sont très longs et où il est moins important de mettre graphiquement en exergue l'enchaînement des jets textuels, l'accent est mis sur la relation entre le NSS et l'organisation des informations textuelles.

4. La transformation de la construction attestée en construction d'identification spécificationnelle permet de confirmer le fonctionnement en tant que NSS d'une occurrence : la nécessité que Kathy puisse effectuer un stage en ESAT $\rightarrow$ la nécessité est que Kathy puisse effectuer un stage en ESAT.

5. Dans les exemples qui suivent, le NSS à analyser est souligné, et la séquence spécifiante est en italique.

6. Voir cependant le travail d'Adler et Legallois (2018) sur les dépendances et attirances dans un corpus de débats parlementaires: "La démarche suivie consiste à calculer, par statistiques, la part que chacune des onze constructions consacre à chaque nom (Attirance), ainsi que la part que chaque nom consacre à chaque construction (Dépendance)." (Adler et Legallois $2018: 1-2$ )

7. Mais nous ne les associerons pas à des types de marqueurs de cohésion, en ne retenant que la vectorialité des dynamiques relationnelles que nous tâcherons d'observer au niveau de l'actualisation des NSS. 
8. "Plus généralement, fait a pour spécificité, contrairement aux autres $\mathrm{N}$ porteurs, de ne pas appliquer de forme conceptuelle au contenu propositionnel qu'il permet de véhiculer." (Huyghe $2018: 38$ )

\section{RÉSUMÉS}

Sémantiquement sous-spécifiés, les noms sous-spécifiés (NSS) sont fonctionnellement très habiles, et assurent des fonctions de connexion ou d'indexation textuelle. Nous nous intéresserons dans cet article à l'actualisation des NSS au cours du processus d'écriture enregistré en temps réel, dans le but d'observer la mise en fonctionnement discursive de cette sous-catégorie nominale et la manière dont les NSS peuvent contribuer à structurer les dynamiques discursives. Nous défendons l'idée selon laquelle les stratégies d'actualisation des NSS au cours du processus d'écriture offrent un éclairage inédit de leur fonctionnement textuel, et permettent aussi d'interroger la notion de sous-spécification, appréhendée dans la dynamique de l'actualisation online et, de ce fait, mise en relation avec la notion de complétion informationnelle.

Semantically under-specified names (NSS) are functionally very skillful, and ensure connection or textual indexing functions. In this paper, we will focus on the production of NSSs during the writing process recorded in real time, in order to observe the discursive functioning of this nominal subcategory and the way it can help to structure discursive dynamics. We defend the idea that the strategies for the NSS production and use during the writing process offer an unprecedented insight into their textual functioning, and also make it possible to question the notion of sub-specification, apprehended in the dynamics of online updating and, as a result, put in relation with the notion of informational completion.

\section{INDEX}

Mots-clés : MoDyCO, Université Paris Nanterre

Keywords : shell-nouns, real-time recorded writing, production, text structuring, connexion, indexation, informational completion 